\title{
How to Build an App without Programming
}

Max Mirho (EntrepreNerd)

\section{KEYWORDS: Educational Services, Product Development, Technology Commercialization.}

\begin{abstract}
A rise in "no-code" tools is letting non-technical founders create technical products without the need to hire a developer.
\end{abstract}

Building an app is hard, right? That's the usual consensus, especially among those who don't have years of experience in programming. Luckily, in the past few years, a rise in "no-code" tools is letting nontechnical founders create technical products without the need to hire a developer. In this article I will be talking about no-code tools that specifically help you build mobile and web apps!

I'll illustrate the power of one of these no-code tools, Adalo (Adalo.com(https://www.adalo.com) ), with an example. Let's say I want to create a platform - a platform where video game players can meet video game creators. The creators need players to test their new games, and the players want to play new games before anyone else. So, we want to create a platform to connect the two groups of individuals!

Before tools like Adalo, to build a two-sided marketplace or platform like this took weeks of development at least. Luckily, Adalo has templates exactly for these kinds of two-sided platforms. To start, Adalo will auto-create login, signup, and profile screens you can customize. You can add your own screens and allow users to select whether they are a player or a game creator, then you customize the "home" screen so it shows information only on the opposite population. Check out the video below to learn how it works.
That might sound a little complicated, but once you play around with Adalo and watch a few tutorials, it makes a ton of sense. It's a visual drag-and-drop editable canvas, and after you get the hang of it, building apps like a two-sided platform are simple. Everything you need is predefined and editable, and half of the time there's going to be an out-of-the-box template for exactly what you need, which could cut your development time down into a matter of hours. (In the video link below, I show you exactly what I mean, and on a basic level, how Adalo works.)

Now, Adalo is an amazing tool, and you can actually launch apps you build on the app store if you want (with the paid plan). But there are simpler tools if you don't need to build something complex. Glide (Glideapps.com(https://www.glideapps.com) ) is a much cheaper tool with simpler functionality that allows you to build phone apps using information contained in a Google Sheet. I was personally able to create a phone app in under 5 minutes (I timed it) that functioned well and had a small chatroom.

Both Adalo and Glide can link up to simple database tools like Airtable and Google Sheets. If you've ever used Microsoft Excel, both of these tools are essentially just online, more advanced versions of the same kind of database. So, if you need data shown in your app, it should be super easy! In fact, Glide MUST be connected to a Google Sheet to function, and the content on your app is built exclusively from that sheet!

\section{Limitations}

These tools will never replace developers. If you need to build something incredibly unique with an intense amount of tech behind it, you're likely not going to be able to complete it with a tool like Adalo or Glide. However, you can create functional prototypes that you can show users and clients incredibly easily. So, even if your technology can't be built fully with no-code, you can build a minimum viable product and test your solution without spending money on an expensive developer.

\section{An Out-of-the-Box Template}


Whether or not you end up fully launching your app idea with Adalo or Glide, using these tools to prototype alone can be incredibly useful. The number one reason startup ventures fail is they build something nobody wants, and that process becomes incredibly risky when you invest time and money into building your product. Instead, start off with a no-code prototype, and test it in the market. If there's no interest whatsoever, then you saved time and money that you would have spent jumping right into expensive product development. Test first, build later.

On that note, tweaking and experimenting in Glide and Adalo is incredibly quick and easy. Whether you've built a full product or a prototype, you'll be able to edit the functionality and even pivot your product entirely to capture new market needs. Being able to rapidly change and evolve your product to capture new needs is necessary to find and solve real problems, and it's a huge advantage in the early stages of building your startup.

Visually though, these apps will look and flow extremely well. You will be shocked, especially by Adalo, with how smooth the interface can be if you know the basics of design. However, few of us are designers, so don't expect it to look perfect or do exactly what you want on the first try. It's going to take some experimentation. If you want to see some popular examples of apps built on these tools, and how wonderfully they work, check out Adalo's showcase page here: https://www.adalo.com/showcase

Finally, aside from my short video above, each tool has a series of tutorials to go along with them and help you get started.

Adalo's Tutorials are here: https://www.adalo.com/videos

Glide's tutorials are

here: https://www.glideapps.com/learn-to-glide

I was able to build a basic app in both without much instruction, but if you want to fully use each tool you should take a few hours to watch their guides. But after that, you're off to the races, and building web apps or mobile apps no longer requires skill with programming. I'm excited to see what you build!
Turn Your Idea into an Innovation (https://eiexchange.com/content/turn-your-idea-into-aninnovation)

\section{Learn More}

